\title{
Detection of very high energy radiation from HESS J1908+063 confirms the Milagro unidentified source MGRO J1908+06
}

F. Aharonian ${ }^{1,13}$, A. G. Akhperjanian ${ }^{2}$, G. Anton ${ }^{16}$, U. Barres de Almeida ${ }^{8, \star}$, A. R. Bazer-Bachi ${ }^{3}$, Y. Becherini ${ }^{12}$, B. Behera ${ }^{14}$, W. Benbow ${ }^{1}$, K. Bernlöhr ${ }^{1,5}$, C. Boisson ${ }^{6}$, A. Bochow ${ }^{1}$, V. Borrel ${ }^{3}$, I. Braun ${ }^{1}$, E. Brion ${ }^{7}$, J. Brucker ${ }^{16}$, P. Brun ${ }^{7}$, R. Bühler ${ }^{1}$, T. Bulik ${ }^{24}$, I. Büsching ${ }^{9}$, T. Boutelier ${ }^{17}$, S. Carrigan ${ }^{1}$, P. M. Chadwick ${ }^{8}$, A. Charbonnier ${ }^{19}$, R. C. G. Chaves ${ }^{1}$, A. Cheesebrough ${ }^{8}$, L.-M. Chounet $^{10}$, A. C. Clapson $^{1}$, G. Coignet $^{11}$, M. Dalton ${ }^{5}$, M. K. Daniel ${ }^{8}$, B. Degrange ${ }^{10}$, C. Deil ${ }^{1}$, H. J. Dickinson ${ }^{8}$, A. Djannati-Atai ${ }^{12}$, W. Domainko ${ }^{1}$, L. O'C. Drury ${ }^{13}$, F. Dubois ${ }^{11}$,

G. Dubus ${ }^{17}$, J. Dyks ${ }^{24}$, M. Dyrda ${ }^{28}$, K. Egberts ${ }^{1}$, D. Emmanoulopoulos ${ }^{14}$, P. Espigat ${ }^{12}$, C. Farnier $^{15}$, F. Feinstein ${ }^{15}$, A. Fiasson ${ }^{15}$, A. Förster ${ }^{1}$, G. Fontaine ${ }^{10}$, M. Füßling ${ }^{5}$, S. Gabici ${ }^{13}$, Y. A. Gallant ${ }^{15}$, L. Gérard ${ }^{12}$, B. Giebels ${ }^{10}$, J. F. Glicenstein ${ }^{7}$, B. Glück ${ }^{16}$, P. Goret ${ }^{7}$, D. Hauser ${ }^{14}$, M. Hauser ${ }^{14}$, S. Heinz ${ }^{16}$, G. Heinzelmann ${ }^{4}$, G. Henri ${ }^{17}$, G. Hermann ${ }^{1}$, J. A. Hinton ${ }^{25}$, A. Hoffmann ${ }^{18}$, W. Hofmann ${ }^{1}$, M. Holleran ${ }^{9}$, S. Hoppe ${ }^{1}$, D. Horns ${ }^{4}$, A. Jacholkowska ${ }^{19}$, O. C. de Jager ${ }^{9}$, I. Jung ${ }^{16}$, K. Katarzyński ${ }^{27}$, U. Katz ${ }^{16}$, S. Kaufmann ${ }^{14}$, E. Kendziorra ${ }^{18}$, M. Kerschhaggl ${ }^{5}$,

D. Khangulyan ${ }^{1}$, B. Khélifi ${ }^{10}$, D. Keogh ${ }^{8}$, Nu. Komin ${ }^{7}$, K. Kosack ${ }^{1}$, G. Lamanna ${ }^{11}$, J.-P. Lenain ${ }^{6}$, T. Lohse ${ }^{5}$, V. Marandon ${ }^{12}$, J.M. Martin ${ }^{6}$, O. Martineau-Huynh ${ }^{19}$, A. Marcowith ${ }^{15}$, D. Maurin ${ }^{19}$, T. J. L. McComb ${ }^{8}$, M. C. Medina ${ }^{6}$, R. Moderski ${ }^{24}$, E. Moulin ${ }^{7}$, M. Naumann-Godo ${ }^{10}$, M. de Naurois ${ }^{19}$, D. Nedbal ${ }^{20}$, D. Nekrassov ${ }^{1}$, J. Niemiec ${ }^{28}$, S. J. Nolan ${ }^{8}$, S. Ohm ${ }^{1}$, J.-F. Olive ${ }^{3}$, E. de Oña Wilhelmi ${ }^{12,29}$, K. J. Orford ${ }^{8}$, M. Ostrowski ${ }^{23}$, M. Panter ${ }^{1}$, M. Paz Arribas $^{5}$, G. Pedaletti ${ }^{14}$, G. Pelletier ${ }^{17}$, P.-O. Petrucci ${ }^{17}$, S. Pita ${ }^{12}$, G. Pühlhofer ${ }^{14}$, M. Punch ${ }^{12}$, A. Quirrenbach ${ }^{14}$, B.C. Raubenheimer ${ }^{9}$, M. Raue ${ }^{1,29}$, S. M. Rayner ${ }^{8}$, M. Renaud ${ }^{1}$, O. Reimer ${ }^{30}$, F. Rieger ${ }^{1,29}$, J. Ripken ${ }^{4}$, L. Rob $^{20}$, S. Rosier-Lees ${ }^{11}$, G. Rowell ${ }^{26}$, B. Rudak ${ }^{24}$, C. B. Rulten ${ }^{8}$, J. Ruppel ${ }^{21}$, V. Sahakian ${ }^{2}$, A. Santangelo ${ }^{18}$, R. Schlickeiser ${ }^{21}$, F. M. Schöck ${ }^{16}$, R. Schröder ${ }^{21}$, U. Schwanke ${ }^{5}$, S. Schwarzburg ${ }^{18}$, S. Schwemmer ${ }^{14}$, A. Shalchi ${ }^{21}$, J. L. Skilton ${ }^{25}$, H. Sol ${ }^{6}$, D. Spangler ${ }^{8}$, Ł. Stawarz ${ }^{23}$, R. Steenkamp ${ }^{22}$, C. Stegmann ${ }^{16}$, G. Superina ${ }^{10}$, P. H. Tam ${ }^{14}$, J.-P. Tavernet ${ }^{19}$, R. Terrier ${ }^{12}$, O. Tibolla ${ }^{14}$, C. van Eldik ${ }^{1}$, G. Vasileiadis ${ }^{15}$, C. Venter ${ }^{9}$, L. Venter ${ }^{6}$, J. P. Vialle ${ }^{11}$, P. Vincent ${ }^{19}$, M. Vivier ${ }^{7}$, H. J. Völk ${ }^{1}$, F. Volpe ${ }^{10,29}$, S. J. Wagner ${ }^{14}$, M. Ward ${ }^{8}$, A. A. Zdziarski ${ }^{24}$, and A. Zech ${ }^{6}$

(Affiliations can be found after the references)

Received 16 November 2008 / Accepted 16 April 2009

\section{ABSTRACT}

Aims. Detection of a $\gamma$-ray source above $300 \mathrm{GeV}$ is reported, confirming the unidentified source MGRO J1908+06, discovered by the Milagro collaboration at a median energy of $20 \mathrm{TeV}$.

Methods. The source was observed during $27 \mathrm{~h}$ as part of the extension of the HESS Galactic plane survey to longitudes $>30^{\circ}$.

Results. HESS J1908+063 is detected at a significance level of $10.9 \sigma$ with an integral flux above $1 \mathrm{TeV}$ of $\left(3.76 \pm 0.29\right.$ stat $\left.\pm 0.75_{\text {sys }}\right) \times$ $10^{-12} \mathrm{ph} \mathrm{cm}^{-2} \mathrm{~s}^{-1}$, and a spectral photon index $\Gamma=2.10 \pm 0.07$ stat $\pm 0.2_{\text {sys. }}$. The positions and fluxes of HESS J1908+063 and MGRO J1908+06 are in good agreement. Possible counterparts at other wavelengths and the origin of the $\gamma$-ray emission are discussed. The nearby unidentified GeV source, GRO J1908+0556 (GeV) which also remains unidentified and the new Fermi pulsar 0FGL J1907.5+0617, may be connected to the $\mathrm{TeV}$ source.

Key words. gamma rays: observations

\section{Introduction}

Very High Energy (VHE) $\gamma$-rays probe sites of particle acceleration to ultra-relativistic energies. Observations with HESS (High Energy Stereoscopic System) in the galactic domain, and, in particular, the Galactic plane survey (GPS) of the central region of the Milky Way $\left(l \simeq-30^{\circ}\right.$ to $30^{\circ}, b \simeq-3^{\circ}$ to $\left.3^{\circ}, 2004-2005\right)$, and its extension to longitudes $l \simeq 30^{\circ}$ to $60^{\circ}$ and $l \simeq 280^{\circ}$ to $330^{\circ}$ during 2005-2007, have resulted in the discovery of more than 40 sources (Aharonian et al. 2005, 2006a, 2008a). A large majority of these are extended sources which renders their identification difficult, except when they exhibit a clear morphology

^ Supported by CAPES Foundation, Ministry of Education of Brazil. or correlation with an identified object at other wavelengths. In addition to firmly identified classes, i.e. shell-type supernova remnants (SNRs), pulsar wind nebulae (PWNe) and X-ray binary systems (XRBs), strong evidence has been obtained for interaction of enhanced fluxes of cosmic rays with target material in the surroundings of SNRs (Aharonian et al. 2008b,c; Albert et al. 2007), as well as in the central 100 pc of the Galaxy (Aharonian et al. 2006b). Nevertheless the majority of galactic $\gamma$-ray sources discovered by HESS still lack firm identification (Aharonian et al. 2008a).

The Milagro collaboration has recently published its sky survey results after seven years of operation (2358 days of data observed from a site at $35^{\circ} \mathrm{N}$ latitude, Abdo et al. 2007), announcing the discovery of three low-latitude sources and 4 lower 
significance hot-spots. One of the sources, MGRO J1908+06, is detected at $8.3 \sigma$ (pre-trial) confidence level, with a differential flux of $(8.8 \pm 2.4$ stat \pm 2.6 sys $) \times 10^{-15} \mathrm{TeV}^{-1} \mathrm{~cm}^{-1} \mathrm{~s}^{-1}$, derived at $\sim 20 \mathrm{TeV}$ assuming a spectral photon index of 2.3. Its angular extension remains unknown, but is bounded to a maximum diameter of $2.6^{\circ}$. MGRO J1908+06 is located near $40^{\circ}$ longitude $\left(l=40^{\circ} 24^{\prime} \pm 6{ }^{\prime} 0_{\text {stat }} \pm 18{ }^{\prime} 0_{\text {sys }}, b=-1^{\circ} 0^{\prime} \pm 6{ }^{\prime} 0_{\text {stat }} \pm 18{ }^{\prime} 0_{\text {sys }}\right)$, which is covered by the extended HESS GPS.

The detection of an extended $\gamma$-ray source, HESS J1908+063, in a compatible direction with that of MGRO J1908+06, is reported here. The lower energy threshold of HESS and its superior angular resolution are used advantageously to derive the position, morphology and the spectrum of the source from 0.3 up to $30 \mathrm{TeV}$. After the description of the observations, data analysis and results in Sect. 2, the HESS source characteristics, given in Sect. 3, will be compared to that of MGRO J1908+06. Its possible counterparts shall finally be discussed in Sect. 4.

\section{Observations and detection of HESS J1908+063}

HESS is an array of four Imaging Atmospheric Cherenkov telescopes and is located in the Khomas Highland in Namibia. Each telescope has a mirror area of $107 \mathrm{~m}^{2}$ (Bernlöhr et al. 2003) and a total field of view of $5^{\circ}$ (Vincent et al. 2003), well suited for the study of extended sources. The system works in a coincidence mode (e.g. Funk et al. 2004), requiring at least two of the four telescopes to have triggered. Its angular resolution reaches $\sim 5^{\prime}$ per event and its sensitivity for a point-like source is $2.0 \times 10^{-13} \mathrm{ph} \mathrm{cm}^{-2} \mathrm{~s}^{-1}$ ( $1 \%$ of the Crab Nebula flux above $1 \mathrm{TeV}$ ) for a $5 \sigma$ detection within $25 \mathrm{~h}$ observation time.

Observations near Galactic longitude $l=40^{\circ}$ were first performed during June 2005 and then from May to September 2006, as part of the extension of the HESS GPS to $l \simeq 30^{\circ}$ to $60^{\circ}$. Subsequent to the first detection of HESS J1908+063, follow-up observations were made in wobble mode during 2007, where the source direction was positioned $\pm 0.5^{\circ}$ in declination relative to the center of the field of view of the camera. Due to the large field of view and uniform response of the HESS cameras, the use of wobble mode allows for both on-source observations and simultaneous estimation of the background induced by charged cosmic rays, since the background can be estimated from different regions in the same field of view. The direction of the offset was alternated in successive scans to reduce systematic effects. Due to the survey-mode observations the source is offset from the field of view center at different angular distances. Observations with an offset of more than $2.0^{\circ}\left(2.5^{\circ}\right)$ were not used for the determination of the spectrum (for the sky maps). The total dead-time corrected, quality and offset selected $\left(2.5^{\circ}\right)$ data set amounts to 27 hours and has an average offset of $1.0^{\circ}$. The zenith angle ranges from 30 to $55^{\circ}$, leading to a mean energy threshold of $\sim 300 \mathrm{GeV}$, for a cut on the image size of 80 photoelectrons (p.e.), and $\sim 600 \mathrm{GeV}$ for a tighter cut of 200 p.e.

After calibration, the standard HESS event reconstruction scheme was applied to the data (Aharonian et al. 2006c). In order to reject the background of cosmic-ray showers, $\gamma$-ray like events were selected using cuts on image shape parameters scaled with their expected values obtained from Monte Carlo simulations. As described in Aharonian et al. (2006a), two different sets of cuts were applied. Cuts optimized for a hard photon spectrum and a weak source with a rather tight cut on the image size of 200 p.e., which achieve a maximum signal-to-noise ratio, were applied to study the morphology of the source, while for the spectral analysis, the image size cut was loosened to 80 p.e. in

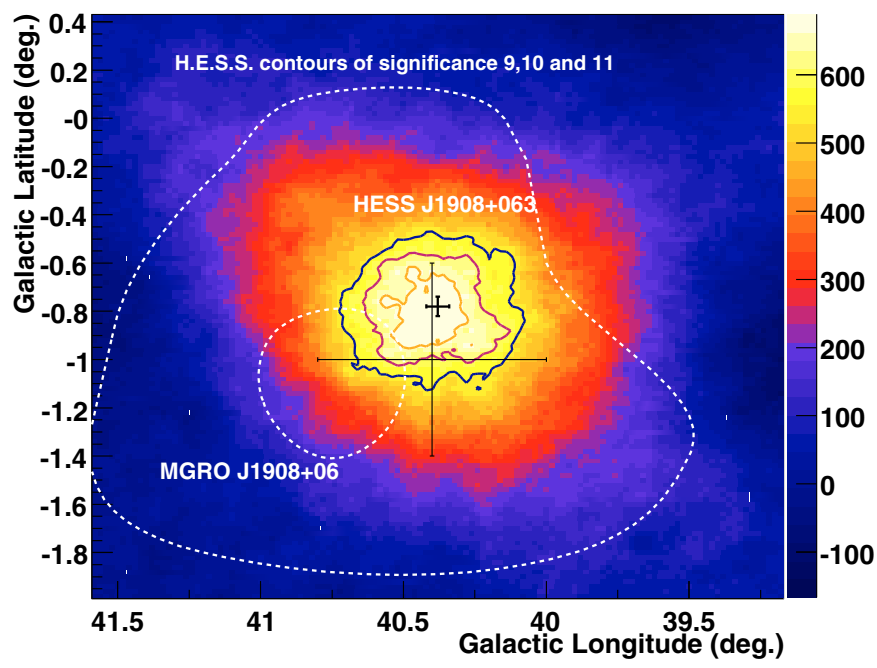

Fig. 1. Gaussian-smoothed $\left(\sigma=0.5^{\circ}\right)$ excess map of the $2.4^{\circ} \times 2.4^{\circ}$ field of view around the position of HESS J1908+063. The colored contours show the pre-trial significance levels for 9, 10 and $11 \sigma$. The dotted white lines show the Milagro significance contours for $8 \sigma$ (inner) and $5 \sigma$ (outer contour). The black crosses mark the best HESS and Milagro fitted positions, respectively, the error bars being the linear sum of the statistical and systematic errors.

order to cover the maximum energy range. The background estimation (described in Berge et al. 2007) for each position in the two-dimensional sky-map was computed from a ring with an (a-priori) increased radius of $1.0^{\circ}$, as compared to the standard radius of $0.7^{\circ}$, in order to account with the large source diameter. The width of the ring was selected to reach a background area four times that of the on source area.

Figure 1 shows the Gaussian-smoothed $\left(\sigma=0.5^{\circ}\right.$ to compare to the Milagro point spread function, PSF) excess map for a size cut on the images above 200 p.e. An excess of 689 events at a pre-trial significance of $12.1 \sigma$ is obtained using an integration radius of $0.5^{\circ}$. A conservative estimate of the trials, following the same procedure as that described in Aharonian et al. (2006a) but taking into account the larger area covered by the extended GPS, yields a post-trial significance of $10.9 \sigma$ (for 525000 trials).

To evaluate the extension and the position of the source, the uncorrelated excess sky-map was fitted to a symmetrical two-dimensional Gaussian function, convolved with the HESS PSF (PSF $\sim 0.08^{\circ}$ above 200 p.e.). The best-fit position lies at $l=40^{\circ} 23^{\prime} 9^{\prime} \cdot 2 \pm 22^{\prime} 4$ stat and $b=-0^{\circ} 47^{\prime} 10^{\prime} .^{\prime} 1 \pm 2{ }^{\prime} .4$ stat, with a systematic error of 20" per axis (Gillessen 2004), and the intrinsic extension derived is $\sigma_{\mathrm{src}}=0.34_{-0.03}^{\circ+0.04}$.

\section{Morphology and spectrum}

To study the morphology of the source, a cut of 200 p.e. on the shower size was applied to optimize the signal-to-background ratio and angular resolution, and the resulting sky map was Gaussian-smoothed $\left(\sigma=0.11^{\circ}\right)$. As can be seen in Fig. 2, the excess event map departs apparently from a Gaussian shape, but the significance of this effect is marginal. When excess events are separated in two different energy bands, 0.7 to $2.5 \mathrm{TeV}$, and that above $2.5 \mathrm{TeV}$ (the two bands were selected (a-priori) such that the signal-to-noise ratio remains constant under the hypothesis of a source with a hard spectrum photon index of 2.2), the emission peaks of the corresponding excess maps show a slight 


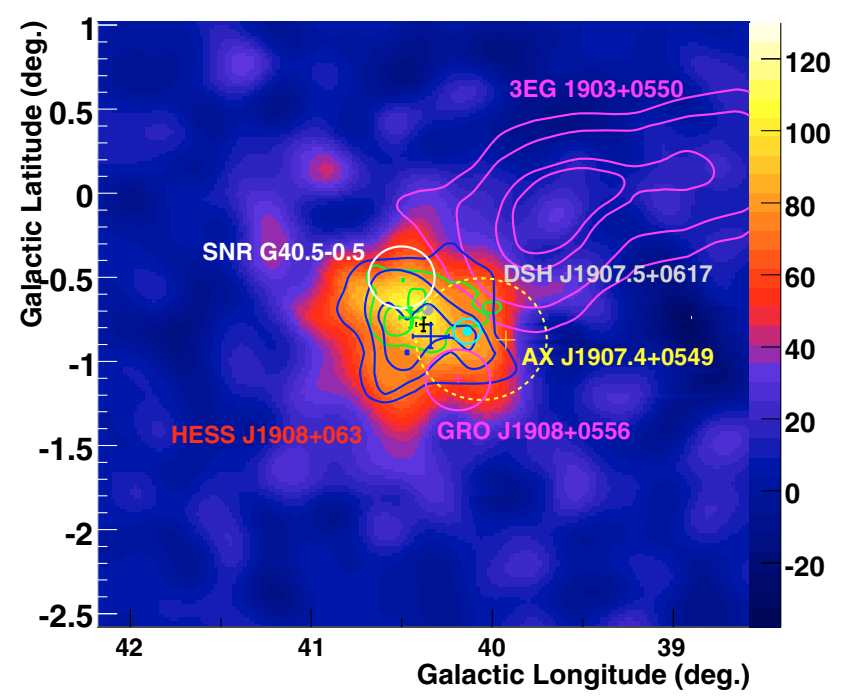

Fig. 2. Gaussian-smoothed $\left(\sigma=0.11^{\circ}\right)$ excess map of the $3.6^{\circ} \times 3.6^{\circ}$ field of view around the position of HESS J1908+063. The HESS significance contours (for an integration radius of $0.22^{\circ}$ ) above $4 \sigma$ are superimposed and marked in green (for the energy range between 0.7 to $2.5 \mathrm{TeV}$ ) and blue (for energies above $2.5 \mathrm{TeV}$ ) contours. The green and blue crosses show the two best fitted positions for the two excesses at the two different energy ranges, the bars being the statistical errors. The possible counterparts are also shown (see Sect. 4.3).

offset $\left(0.16^{\circ}\right.$ apart $)$ with respect to each other, following roughly the morphology of the total excess map.

Two circular regions of radius $0.2^{\circ}$ were selected around the positions of the low and high energy fitted centroids. The energy spectrum was derived in these two regions (see below for details of the procedure). The results of these spectral analysis show the expected tendency, i.e. the spectral index derived at the high energy peak is harder than the one at the low energy region, with a difference of 0.17 . Nevertheless the systematic error of the measurements $( \pm 0.2)$ forbids any conclusion on the separation in two sources/peaks.

The energy spectrum was derived considering a single source within an integration radius of $0.5^{\circ}$ (to take into account the angular extension of the source), centered on the best-fit position, by means of a forward-folding maximum likelihood fit (Piron et al. 2002). The background was evaluated from positions in the field of view with the same radius and same offset from the pointing direction as the source region. The spectrum, shown in Fig. 3, is well fitted with a simple power-law function with a hard photon index of $2.10 \pm 0.07$ stat $\pm 0.2_{\text {sys }}$ and a differential flux at $1 \mathrm{TeV}$ of $\left(4.14 \pm 0.32\right.$ stat $\left.\pm 0.83_{\text {sys }}\right) \times 10^{-12} \mathrm{TeV}^{-1} \mathrm{~cm}^{-2} \mathrm{~s}^{-1}$. The systematic error on the flux is conservatively estimated from simulated data to be $20 \%$ while the photon index has a typical systematic error of \pm 0.2 . The integrated flux above $1 \mathrm{TeV}$ corresponds to $17 \%$ of the Crab Nebula flux above that energy (Aharonian et al. 2006c).

An exponential energy cut-off has been searched for in the data. The flux was fit by a power law with an exponential cutoff of the form $F(E)=N_{\mathrm{o}}(\mathrm{E} / 1 \mathrm{TeV})^{-\Gamma} \exp \left(E / E_{\text {cut }}\right)$, where $N_{\mathrm{o}}$ is the flux normalization and $\Gamma$ the photon index. There is no indication for such a cut-off and a lower limit of $E_{\text {cut }}>19.1 \mathrm{TeV}( \pm 15 \%$ systematic error) can be derived at $90 \%$ confidence level.

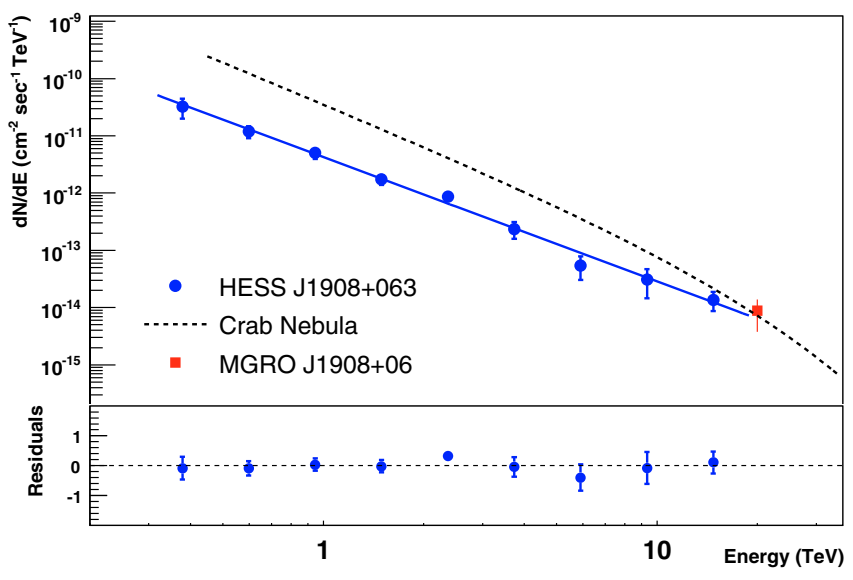

Fig. 3. Differential energy spectrum of HESS J1908+063 measured in the energy range $300 \mathrm{GeV}-30 \mathrm{TeV}$. The differential flux of MGRO J1908+06 at $20 \mathrm{TeV}$ is shown in red. The black dotted line represents the Crab Nebula energy spectrum measured by HESS. The residuals to the power-law fit are shown in the lower panel.

\section{Discussion}

\subsection{Comparison with MGRO J1908+06}

The fitted positions of HESS J1908+063 and MGRO J1908+06 and their significance contours are in good agreement as shown in Fig. 1. The association is supported by a comparison of the HESS derived spectrum to the Milagro differential flux at $20 \mathrm{TeV}$ (see Fig. 3), where the Milagro point error bar is the linear sum of the statistical and systematic errors while the HESS points error bars represent only the statistical error. The latter was obtained using a larger integration radius of $1.3^{\circ}$ (90\% CL), compared to $0.5^{\circ}$ for HESS $\mathrm{J} 1908+063$. The positional and flux agreements (the systematic errors on absolute flux measurements are about $20-30 \%$ for the two instruments) are consistent with the fact that the emission seen in the two instruments comes from the same source, and that no other significant emission from outside the HESS integration radius of $0.5^{\circ}$ contributes to the flux of MGRO J1908+06.

\subsection{Milagro detectability - neighbouring HESS sources}

Since the detection of HESS J1908+063 confirms for the first time with a Cherenkov telescope one of the Milagro sources, the case of other HESS sources at galactic longitudes to the North of $30^{\circ}$, which therefore fall into the overlap region with the Milagro sky survey, is relevant in this context. These are HESS J1857+026, formerly unidentified but for which a potential counterpart, PSR J1856+0245, has been recently discovered (Aharonian et al. 2008a; Hessels et al. 2008) the still unidentified HESS J1858+020 (Aharonian et al. 2008a), and the PWN candidate HESS J1912+101 (Aharonian et al. 2008d).

In order to investigate if these sources were susceptible to be detected by Milagro, their differential flux, extrapolated to $20 \mathrm{TeV}$, is compared to the Milagro sensitivity at ecliptic declinations of $0^{\circ}$ and $10^{\circ}$ (taken from Abdo et al. 2007) in Fig. 4. HESS J1857+026, with a much smaller angular extension $\left(\sim 0.11^{\circ}\right)$, a higher differential flux at $1 \mathrm{TeV}(6.1 \times$ $\left.10^{-12} \mathrm{TeV}^{-1} \mathrm{~cm}^{-2} \mathrm{~s}^{-1}\right)$ but a slightly softer spectrum $(2.39 \pm$ 0.08 stat), seems at the limit of detectability, while the two others appear to be well below the Milagro sensitivity. The spectral 


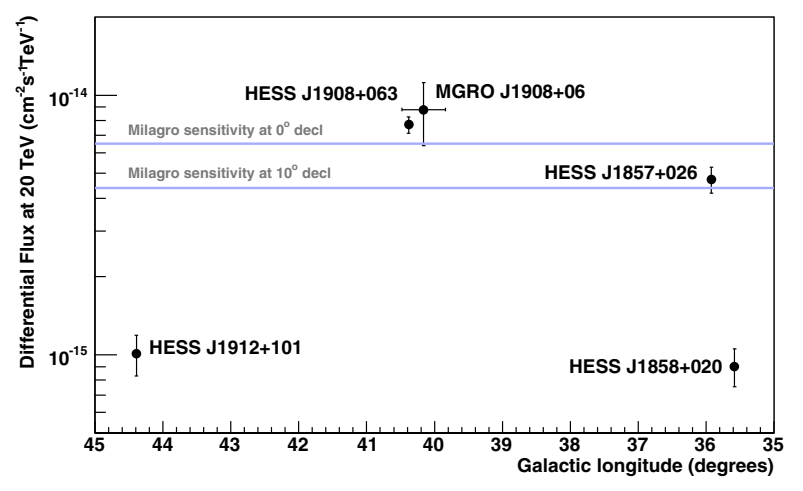

Fig. 4. Differential flux at $20 \mathrm{TeV}$ vs. galactic longitude for sources detected by HESS and Milagro for $l \in\left[35^{\circ}, 45^{\circ}\right]$. The blue lines show the differential sensitivity of Milagro for $0^{\circ}$ and $10^{\circ}$ declination, which correspond to the declination range of Milagro observations for these galactic longitude values.

index of HESS J1858+020 is hard $(2.17 \pm 0.12$ stat $)$, but its differential flux is an order of magnitude below the other sources. HESS J1912+101, is closer to HESS J1908+063 in angular extension $\left(0.26^{\circ}\right)$ with a comparable differential flux at $1 \mathrm{TeV}$ $\left(\sim 3.5 \times 10^{-12} \mathrm{TeV}^{-1} \mathrm{~cm}^{-2} \mathrm{~s}^{-1}\right)$, but has a much steeper spectrum index $(2.7 \pm 0.2$ stat $)$. This explains the dramatic drop in its differential flux at $20 \mathrm{TeV}$ as compared to HESS J1908+063.

\subsection{Search for counterparts}

Potential counterparts for HESS J1908+063 are shown in Fig. 2: the Galactic open cluster candidate DSH J1907.5+0617, marked with a grey point; the SNR G40.5-0.5, whose angular extension is marked with a white circle; the EGRET source 3EG 1903+0550, in purple contours corresponding to 99, 95, 68 and $50 \%$ confidence levels and considered as possibly associated with the SNR (Hartman et al. 1999); the ASCA source AX J1907.4+0549, shown in light yellow (the dotted circle shows its observation field of view), was discovered as a result of the systematic search for identification of EGRET sources (Roberts et al. 2001) and was proposed as a plausible counterpart to the other yet unidentified EGRET source in the field, GRO J1908+0556 (GeV) (Reimer et al. 1997; Lamb et al. 1997); the latter is marked with a cross in purple together with its $1 \sigma$ position error. Recently a new pulsar, 0FGL J1907.5+0602, detected by the Fermi-LAT instrument (Abdo et al. 2009a) was reported (cyan circle) close to both the TeV and EGRET sources.

The 26' shell SNR G40.5-0.5 is in spatial coincidence with the northeastern part of the HESS source. Downes et al. (1980), using various $\Sigma-D$ relations derived a linear diameter of $40-65 \mathrm{pc}$, an age of $(2-4) \times 10^{4} \mathrm{yr}$, and estimated the distance to the SNR to be in the range 5.5 to $8.5 \mathrm{kpc}$, corresponding to a location either in the inter-arm region between the Scutum and the Sagittarius arms, or on the inner edge of the latter. Yang et al. (2006) investigated the distribution of molecular gas around the SNR direction through the ${ }^{12} \mathrm{CO}(J=1-0)$ transition line, and provided evidence for interaction between the SNR and its neighboring dense ISM at a central velocity $V_{\mathrm{LSR}}=55 \mathrm{~km} \mathrm{~s}^{-1}$. The corresponding kinematic distance of 3-3.4 kpc implied a source diameter of $25 \mathrm{pc}$ and a younger age, at variance with the initial estimates of Downes et al. (1980).

The association of the HESS source to this SNR is not straightforward due to the fact that the angular size $\left(>40^{\prime}\right.$

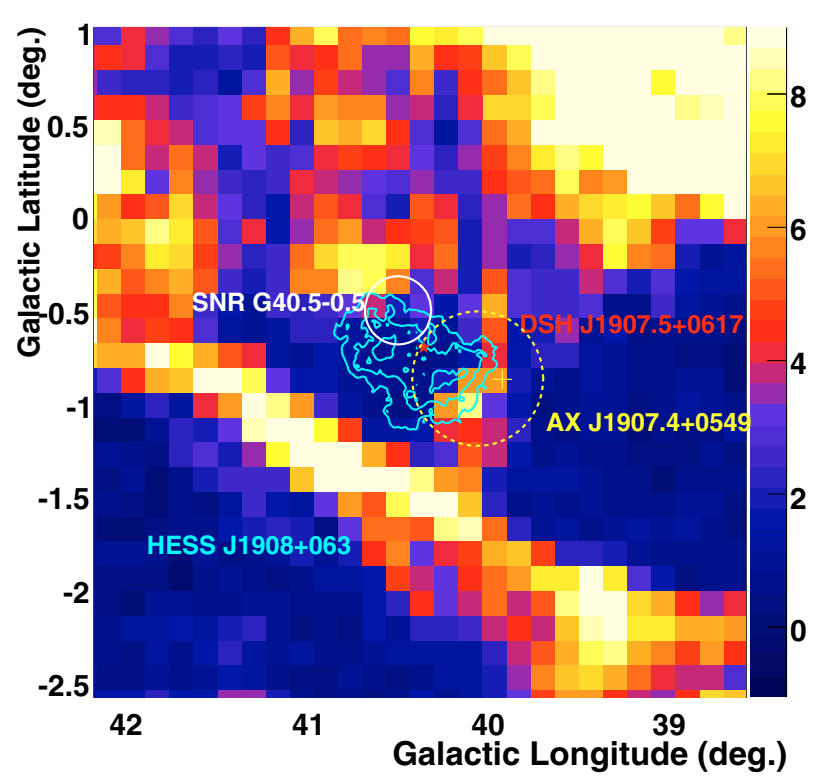

Fig. 5. On the color scale background, the sky-map in ${ }^{12} \mathrm{CO}(J=1-0)$ integrated in the velocity range between 25.3 and $30.5 \mathrm{~km} \mathrm{~s}^{-1}$ around the position of the HESS source, in blue contours. Possible counterparts are also shown (see Sect. 4.3).

$F W H M$ ) of the VHE source is significantly larger than the $26^{\prime}$ size of the shell. A scenario consisting of a SNR-molecular cloud association, such as that possibly at work for SNR W 28 (Aharonian et al. 2008b) where the $\gamma$-rays are produced through interactions of accelerated cosmic rays with molecular matter in the vicinity of the source, along with the contribution of a nearby unresolved source to the south-west could lead to such a difference in angular extension. However, the dense region reported by Yang et al. (2006) is located north of the SNR and does not correspond to the observed $\gamma$-ray emission.

The study of the ${ }^{12} \mathrm{CO}(J=1-0)$ data (Dame et al. 2001) in the line of sight of HESS J1908+063 confirms this discrepancy. However, in the velocity range $\mathrm{V}_{\mathrm{LSR}}=25-30 \mathrm{~km} \mathrm{~s}^{-1}$, corresponding to a much closer distance of $1.5-1.8 \mathrm{kpc}-$ that is the closer part of the Sagittarius arm- an interesting feature might be present: the VHE source is aligned with a very low density region resembling a bubble left by a supernova explosion (Fig. 5) or stellar winds. Kronberger et al. (2006) reported a candidate for a Galactic open cluster, DSH J1907.5+0617, whose position is compatible with the HESS source best fitted one. Deeper studies of this candidate are necessary to further clarify its nature and establish a possible connection with the VHE source and ${ }^{12} \mathrm{CO}$ $(J=1-0)$ data.

The 2-10 keV ASCA GIS image, which led to the discovery of AX J1907.4+0549, partially covers the field of view of the HESS source. Another X-ray observation with Swift/XRT was recently reported (Kong 2007), following the announcement of a faint radio source in archival data (Combi et al. 2007) near the HESS J1908+063 centroid $\left(\mathrm{RA}=19^{\mathrm{h}} 08^{\mathrm{m}} 03.73^{\mathrm{s}}\right.$, Dec $=+06^{\circ} 18^{\prime} 22^{\prime \prime}$. 4 ), but resulted only in a $3 \sigma$ upper-limit for a point-like source on the unabsorbed $0.3-10 \mathrm{keV}$ flux of $2.8 \times$ $10^{-13} \mathrm{erg} \mathrm{cm}^{-2} \mathrm{~s}^{-1}$. AX J1907.4+0549 consists of two unresolved peaks, possibly due to a diffuse emission (Roberts et al. 2001), and lies at the western edge of HESS J1908+063, $0.33^{\circ}$ to its fitted centroid. If the X-ray source is indeed extended, it could be a PWN candidate, although up to now no pulsar has 
been found in this direction (Roberts et al. 2002). Assuming there is a PWN in X-rays, its association to the VHE source would imply an angular offset of $0.48^{\circ}$. This type of morphology is not unusual: other PWNe associations such as HESS J1825137, MSH 15-52, HESS J1718-385, etc. (see e.g. Lemiere et al. 2007) exhibit such a configuration which is explained either by the expansion of the SNR in an inhomogeneous medium and/or the proper motion of the pulsar (see e.g. Blondin et al. 2001). Nevertheless the ASCA X-ray data statistics is too scarce to establish the existence of a PWN on its own.

On the other hand, the recently discovered Fermi-LAT pulsar, OFGL $\mathrm{J} 1907.5+0602$, lies at an offset of $0.26^{\circ}$ relative to the HESS source fitted centroid and is likely to be associated with it; if so, this would also imply an asymmetrical PWN morphology. Previously, in the same energy range, two unidentified EGRET objects were found in the vicinity of HESS J1908+063. The harder source, GRO J1908+0556 $(\mathrm{GeV})$, or alternatively GeV J1907+0557 (the positions of the two sources are compatible within errors), is consistent with the position of the FermiLAT source and lies within a compatible distance to the centroid of the HESS source, roughly two times the EGRET $68 \%$ position measurement error. If the $\mathrm{TeV}$ source and the Fermi pulsar are indeed related, a sharp cutoff in the photon spectrum of the latter is expected and no contribution at $\mathrm{TeV}$ energies is expected. The integral flux $>1 \mathrm{GeV}$ reported for GRO J1908+0556 (GeV) $\left(6.33 \times 10^{-8} \mathrm{ph} \mathrm{cm}^{-2} \mathrm{~s}^{-1}\right)$ is almost twice that of the Fermi source $\left(3.74 \times 10^{-8} \mathrm{ph} \mathrm{cm}^{-2} \mathrm{~s}^{-1}\right)$. If the GRO and Fermi sources are associated together, the larger flux of the former could be due to unpulsed PWN emission, although EGRET calibration issues at several GeV, outlined recently (Abdo et al. 2009b), may have lead to an overestimation of its flux. Also, a naive extrapolation of the HESS spectrum to lower energies $(>1 \mathrm{GeV})$ leads to a lower flux $\left(7.5 \times 10^{-9} \mathrm{ph} \mathrm{cm}^{-2} \mathrm{~s}^{-1}\right)$ than the flux difference between the EGRET source and the Fermi pulsar. Nonetheless the association of the VHE source to either the EGRET GeV source and/or the Fermi pulsar remains likely.

\subsection{Origin of the VHE emission}

$\mathrm{TeV} \gamma$-ray emission manifests the presence of ultra-relativistic particles and could be produced either through Inverse Compton (IC) scattering of the CMBR, IR and/or star-light seed photons by electrons, or from the decay of neutral pions resulting from proton-proton (and other nuclei) interactions. The fact that the spectrum of HESS J1908+063 extends up to energies of at least $\mathrm{E}_{\gamma}=19 \mathrm{TeV}$, supported by the Milagro detection, implies the presence of either electrons of energy $E_{\mathrm{e}} \simeq 18 \times E_{\gamma}^{1 / 2} \simeq 80 \mathrm{TeV}$ (if neglecting the $\sim 30 \%$ drop in the cross section due to the Klein-Nishina effect), or protons up to $E_{\mathrm{p}} \simeq 10 \times E_{\gamma} \sim 200 \mathrm{TeV}$ (for typical proton spectra, see e.g. Kelner et al. 2008). The available multi-wavelength data do not allow to distinguish the nature of the particles at the origin of the $\gamma$-ray emission yet. The X-ray upper-limit of $2.8 \times 10^{-13} \mathrm{erg} \mathrm{cm}^{-2} \mathrm{~s}^{-1}$ (Kong 2007) is calculated for a point-like source, and given the large size of the VHE source, it does not allow to constrain the magnetic field strength for a leptonic scenario. If one assumes the association with the Fermi pulsar and an IC emission by electrons, the extension of the spectrum up to $19 \mathrm{TeV}$ would imply a PWN age of 5-10 kyr for a magnetic field in the 5 to $3 \mu \mathrm{G}$ range (see e.g., Eq. (6) in de Jager \& Djannati-Atai 2009). On the other hand, given that the extrapolation of the HESS source spectrum to lower energies is well below the EGRET source flux, a continuity of the spectral energy distribution from $\mathrm{GeV}$ to tens of $\mathrm{TeV}$ remains possible, in which case a hadronic origin of the $\gamma$-ray emission would constitute also a plausible scenario (Funk et al. 2008).

\section{Conclusions}

A new HESS source, HESS J1908+063, has been detected above $300 \mathrm{GeV}$ at a post-trial significance of $10.9 \sigma$ during the extended HESS Galactic plane survey. It is rather a bright source, with a flux of $17 \%$ of the Crab Nebula. It has a large angular size of $\sigma=0.34^{\circ}$ and shows a hard spectrum with a photon index of $2.10 \pm 0.07_{\text {stat }} \pm 0.2_{\text {sys }}$. The centroid position and the flux of HESS J1908+063 are compatible with those of the unidentified source, MGRO J1908+06, reported by the Milagro collaboration at the median energy of $20 \mathrm{TeV}$. The two sources can hence be considered as identical. For the first time, one of the sources discovered by Milagro is confirmed by an Imaging Atmospheric Cherenkov telescope. The comparison of the fluxes of HESS sources which are covered by the Milagro sky survey to the latter's sensitivity shows that only another source, HESS J1857+026, is at the Milagro detection limit. The hard spectrum of HESS J1908+063 combined with the detection by Milagro of MGRO J1908+06 at median energies of $20 \mathrm{TeV}$ imply the presence of either electrons, or protons, up to 80 or $200 \mathrm{TeV}$, respectively.

The association with SNR G40.5-0.5 to the north-east is not excluded but the larger angular extension of the TeV emission should then find an explanation in terms of either the contribution of unresolved sources or the interactions of ultra-relativistic particles with molecular matter in the vicinity of the SNR. The open cluster candidate DSH J1907.4+0549 is positionally compatible with the HESS source position, and could be possibly associated to a "void" in the ${ }^{12} \mathrm{CO}(J=1-0)$ data. If better measurements support the association to the open cluster, another step could be made towards establishing this class of objects as VHE emitters (Aharonian et al. 2007).

On the other hand, the recently reported $\gamma$-ray pulsar, 0FGL J1907.5+0602, lies close to HESS J1908+063 centroid $\left(0.26^{\circ}\right)$, and could well be associated to the latter in an offsettype PWN scenario, if the system age is of order of 10000 years. The unidentified GeV source GRO J1908+0556 (GeV) lies also at a compatible position to those of the TeV source and the Fermi pulsar and hence could be related to both. The measurement of the unpulsed $\mathrm{GeV}$ component as well as further multiwavelength observations are critical for investigating such association and the nature of HESS J1908+063/MGRO J1908+06.

Acknowledgements. The support of the Namibian authorities and of the University of Namibia in facilitating the construction and operation of HESS is gratefully acknowledged, as is the support by the German Ministry for Education and Research (BMBF), the Max Planck Society, the French Ministry for Research, the CNRS-IN2P3 and the Astroparticle Interdisciplinary Programme of the CNRS, the U.K. Science and Technology Facilities Council (STFC), the IPNP of the Charles University, the Polish Ministry of Science and Higher Education, the South African Department of Science and Technology and National Research Foundation, and by the University of Namibia. We appreciate the excellent work of the technical support staff in Berlin, Durham, Hamburg, Heidelberg, Palaiseau, Paris, Saclay, and in Namibia in the construction and operation of the equipment.

\section{References}

Abdo, A. A., et al. (Fermi Collaboration) 2009a, submitted to ApJS [arXiv: 0902.1340]

Abdo, A. A., et al. (Fermi Collaboration) 2009b, accepted in ApJ [arXiv:0812.2960v1]

Abdo, A. A., Allen, B., Berley, D., et al. (MILAGRO Collaboration) 2007, ApJ, 664,91 
Aharonian, F., Akhperjanian, A. G., Bazer-Bachi, A. R., et al. (HESS Collaboration) 2005, Science, 307, 1938

Aharonian, F., Akhperjanian, A. G., Bazer-Bachi, A. R., et al. (HESS Collaboration) 2006a, ApJ, 636, 777

Aharonian, F., et al. (HESS Collaboration) 2006b, Nature, 432, 75

Aharonian, F., Akhperjanian, A. G., Bazer-Bachi, A. R., et al. (HESS Collaboration) 2006c, A\&A, 457, 899

Aharonian, F., Akhperjanian, A. G., Bazer-Bachi, A. R., et al. (HESS Collaboration) 2007, A\&A, 467, 1075

Aharonian, F., Akhperjanian, A. G., Barres de Almeida, U., et al. (HESS Collaboration) 2008a, A\&A, 477, 353

Aharonian, F., Akhperjanian, A. G., Bazer-Bachi, A. R., et al. (HESS Collaboration) 2008b, A\&A, 481, 401

Aharonian, F., Akhperjanian, A. G., Barres de Almeida, U., et al. (HESS Collaboration) 2008c, A\&A, 490, 685

Aharonian, F., Akhperjanian, A. G., Barres de Almeida, U., et al. (HESS Collaboration) 2008d, A\&A, 484, 435

Albert, J., Aliu, E., Anderhub, H., et al. (MAGIC Collaboration) 2007, ApJ, 664, L87

Berge, D., Funk, S., \& Hinton, J. A. 2007, A\&A, 466, 1219

Bernlöhr, K., Carrol, O., Cornils, R., et al. 2003, APh, 20, 111

Blondin, J. M., Chevalier, R. A., \& Frierson, D. M. 2001, ApJ, 563, 806

Combi, J. A., et al. 2007, The Astronomer's Telegram, 1247

Dame, T. M., Hartmann, D., \& Thaddeus, P. 2001, ApJ, 547, 792

de Jager, O. C., \& Djannati-Atai, A. 2009, Neutron Stars and Pulsars, ed. W. Becker (Springer ASSL), XV, 357, 451

Downes, A. J. B., Pauls, T., \& Salter, C. J. 1980, A\&A, 92, 47

Funk, S., Hermann, G., Hinton, J., et al. 2004, APh, 22, 285

Funk, S., Reimer, O., Torres, D. F., Hinton, J. A., et al. 2008, ApJ, 679, 1299

Gillessen, S. 2004, Ph.D. Thesis, University of Heidelberg

Hartman, R. C., Bertsch, D. L., Bloom, S. D., et al. 1999, AJSS, 123, 1, 79

Hessels, J. W. T., et al. 2008, submitted to ApJL [arXiv:0806.1200v1]

Kelner, S. R., Aharonian, F., \& Bugayov, V. V. 2006, Phys. Rev. D 74, 034018

Kong, A. K. H. 2007, The Astronomer's Telegram, 1251

Kronberger, M., Teutsch, P., Alessi, B., et al. 2006, A\&A, 447, 921

Lamb, R. C., \& Macomb, D. J. 1997, ApJ, 488, 872

Lemiere, A. et al. (HESS Collaboration) 2007, 30th ICRC, Merida [arXiv:0710.3528v1]

Piron, F., Djannati-Ataï, A., Punch, M., et al. 2001, A\&A, 374, 895

Reimer, O., et al. 1997, In Proceedings 25th ICRC, Durban, 3, 97

Roberts, M. S. E., Romani, R. W., Kawai, N., et al. 2001, ApJS, 133, 451

Roberts, M. S. E., Hessels, J. W. T., Ransom, S. M., et al. 2002, ApJ, 577, L19

Vincent, P., et al. 2003, in Proceedings of the 28th International Cosmic Ray Conference, Tsukuba, 1, 2887

Yang, J., Zhang, J.-L., Cai, Z. Y., Lu, D.-R., \& Tan, Y.-H. 2006, Ch. Journ. A\&A, 6,210

1 Max-Planck-Institut für Kernphysik, PO Box 103980, 69029

Heidelberg, Germany

2 Yerevan Physics Institute, 2 Alikhanian Brothers St., 375036

Yerevan, Armenia

3 Centre d'Étude Spatiale des Rayonnements, CNRS/UPS, 9 av. du

Colonel Roche, BP 4346, 31029 Toulouse Cedex 4, France

4 Universität Hamburg, Institut für Experimentalphysik, Luruper

Chaussee 149, 22761 Hamburg, Germany

5 Institut für Physik, Humboldt-Universität zu Berlin, Newtonstr. 15, 12489 Berlin, Germany

6 LUTH, Observatoire de Paris, CNRS, Université Paris Diderot, 5 place Jules Janssen, 92190 Meudon, France
7 IRFU/DSM/CEA, CE Saclay, 91191 Gif-sur-Yvette, Cedex, France

8 University of Durham, Department of Physics, South Road, Durham DH1 3LE, UK

9 Unit for Space Physics, North-West University, Potchefstroom 2520, South Africa

${ }^{10}$ Laboratoire Leprince-Ringuet, École Polytechnique, CNRS/IN2P3, 91128 Palaiseau, France

11 Laboratoire d'Annecy-le-Vieux de Physique des Particules, CNRS/IN2P3, 9 chemin de Bellevue, BP 110, 74941 Annecy-leVieux Cedex, France

12 Astroparticule et Cosmologie (APC), CNRS, Université Paris 7 Denis Diderot, 10 rue Alice Domon et Léonie Duquet, 75205 Paris Cedex 13, France (UMR 7164, CNRS, Université Paris VII, CEA, Observatoire de Paris, France)

e-mail: emma@apc.univ-paris7.fr

e-mail: djannati@apc . univ-paris7.fr

13 Dublin Institute for Advanced Studies, 5 Merrion Square, Dublin 2, Ireland

${ }^{14}$ Landessternwarte, Universität Heidelberg, Königstuhl, 69117 Heidelberg, Germany

15 Laboratoire de Physique Théorique et Astroparticules, CNRS/IN2P3, Université Montpellier II, CC 70, Place Eugène Bataillon, 34095 Montpellier Cedex 5, France

16 Universität Erlangen-Nürnberg, Physikalisches Institut, Erwin-Rommel-Str. 1, 91058 Erlangen, Germany

17 Laboratoire d'Astrophysique de Grenoble, INSU/CNRS, Université Joseph Fourier, BP 53, 38041 Grenoble Cedex 9, France

18 Institut für Astronomie und Astrophysik, Universität Tübingen, Sand 1, 72076 Tübingen, Germany

19 LPNHE, Université Pierre et Marie Curie Paris 6, Université Denis Diderot Paris 7, CNRS/IN2P3, 4 place Jussieu, 75252 Paris Cedex 5, France

${ }^{20}$ Institute of Particle and Nuclear Physics, Charles University, V Holesovickach 2, 18000 Prague 8, Czech Republic

${ }^{21}$ Institut für Theoretische Physik, Lehrstuhl IV: Weltraum und Astrophysik, Ruhr-Universität Bochum, 44780 Bochum, Germany

${ }^{22}$ University of Namibia, Private Bag 13301, Windhoek, Namibia

23 Obserwatorium Astronomiczne, Uniwersytet Jagielloński, ul. Orla 171, 30-244 Kraków, Poland

${ }^{24}$ Nicolaus Copernicus Astronomical Center, ul. Bartycka 18, 00716 Warsaw, Poland

25 School of Physics \& Astronomy, University of Leeds, Leeds LS2 9JT, UK

26 School of Chemistry \& Physics, University of Adelaide, Adelaide 5005, Australia

27 Torun Centre for Astronomy, Nicolaus Copernicus University, ul. Gagarina 11, 87-100 Torun, Poland

28 Instytut Fizyki Jạdrowej PAN, ul. Radzikowskiego 152, 31-342 Kraków, Poland

29 European Associated Laboratory for Gamma-Ray Astronomy, jointly supported by CNRS and MPG

30 Stanford University, HEPL \& KIPAC, Stanford, CA 94305-4085, USA 